\section{Records of digger wasps from Eastern Siberia and the Far East of Russia (Hymenoptera: Sphecidae, Crabronidae)}

With 8 figures and 2 maps

HANS-JOACHIM JACOBS ${ }^{1,2}$ and WOLF-HARALD LIEBIG ${ }^{3}$

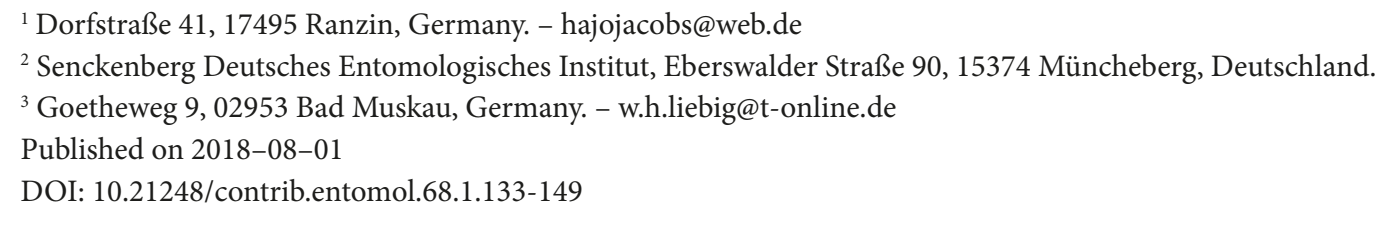

\section{Abstract}

Information is given on the distribution of 17 species of Sphecidae and 106 species of Crabronidae in Eastern Siberia and the Russian Far East. First records for Eastern Siberia are Crossocerus (Crossocerus) tarsatus (SHuCKARD, 1837), Diodontus handlirschi KoHL, 1888, Gorytes albidulus (Lepeletier, 1832), Gorytes procrustes HaNdLIRSCH, 1888, Nysson interruptus (FABRICIUs, 1798), Oxybelus haemorrhoidalis OLIVIER, 1812 and Pemphredon lethifer (SHUCKARD, 1837). First recorded from Far East is Ectemnius (Ectemnius) guttatus (VANDER Linden, 1829).

\section{Zusammenfassung}

Informationen zur Verbreitung von 17 Arten der Sphecidae und 106 Arten der Crabronidae im Osten Sibiriens werden präsentiert. Erstfunde für Ostsibirien sind Crossocerus (Crossocerus) tarsatus (SHUCKARD, 1837), Diodontus handlirschi Kohl, 1888, Gorytes albidulus (Lepeletier, 1832), Gorytes procrustes Handlirsch, 1888, Nysson interruptus (FABricius, 1798), Oxybelus haemorrhoidalis Olivier, 1812 und Pemphredon lethifer (ShuCKard, 1837). Erstfund für den Fernen Osten ist Ectemnius (Ectemnius) guttatus (VANDER Linden, 1829).

\section{Key words}

Insecta, Hymenoptera, Sphecidae, Crabronidae, Distribution, Russia, Siberia, Far East

\section{Introduction}

The digger wasp fauna of Siberia and the Far East of Russia has moved more into the focus of entomologists during recent years. Wolf-Harald Liebig carried out four expeditions to Eastern Siberia and the Far East. Two of these excursions (2007 and 2014) examined the western banks of Lake Baikal (Irkutsk Province), another (2012) Buryat Republic, and the last (2014) Primorskii Territory. Most records presented here resulted from these expeditions. Supplementary records are from material in the collection of The Bavarian State Collection of Zoology Munich (Zoologische Staatssammlung München) (mainly specimens from the island Sakhalin), the collection of Senckenberg Deutsches Entomologisches Institut, and the private collections of C. Schmid-Egger (Berlin) and M. Jänicke (Eisenberg). 


\section{Material and methods}

For the present study 892 specimens (162 Sphecidae, 730 Crabronidae) were examined. Determination of most specimens was undertaken with the key by NemKov et al. (1995). Additionally, the keys by Bouček (2001), Danilov (2014b), Dollfuss (2010, 2013), Gayubo \& Felton (2000), Jacobs (2007), Kohl (1906), Marshakov (1979), Nemkov (1996) and Schmid-Egger (2016) were used. For identification of Gorytes Latreille, 1805 the keys by Nemkov $(1989,1990)$ and Schmid-Egger (2002) were used. However, the identification of some males proved to be problematic. According to SCHMID-EgGER (personal communication), some "European" taxa from the Far East represent separate species. This hypothesis can only be confirmed by revision of the Palaearctic species. The distribution of digger wasps in Eastern Siberia is treated in the catalogues of Nemкov (2008, 2009, 2012a), DANilov (2014a) and Belokobylskij \& Lelej (2017) if not mentioned otherwise. Distribution data of all species here listed are presented in the catalogue of BELOKOBYLSKIJ \& LeLEj (2017). Records from Russia are given for the main geographic parts as treated by BELOKOBYLSKIJ \& LELEJ (2017). Distribution in the provinces may check in the catalogue. New local records from the Russian provinces are marked with an asterisk $\left(^{\star}\right)$.

Specimens are stored in coll. Liebig (Bad Muskau) and coll. Jacobs (Ranzin), in the coll. of The Bavarian State Collection of Zoology Munich (leg. Nesterov, Golovushkin), coll. Schmid-Egger (Berlin) (leg. Lelej, Mattes and some specimens leg. Nesterov) and the coll. Jänicke (Eisenberg) (leg. Jänicke). Specimens collected by Taeger are stored in the coll. of Senckenberg Deutsches Entomologisches Institut Müncheberg. The maps were constructed with help of Google Earth Pro () 2017 Google, (c) 2017 ZENRIN, Image Landsat / Copernicus, US Dept. of State Geographer). The coordinates are in decimal degrees. Collecting dates are in German format, as on the collecting labels. Especially elder collecting labels bears no coordinates. Coordinates of this collecting sites are completed by the authors. Maybe, it isn't the real collecting place sometimes, but the next settlement.

\section{Index of localities}

Eastern Siberia, Tuva Republic (Fig. 1, Map 1). Kysyl, about $700 \mathrm{~m}$ a.s.l. $\left(51.71^{\circ} \mathrm{N} 94.45^{\circ} \mathrm{E}\right)$. Eastern Siberia, Irkutsk Province (Fig. 1, Map 1). Angarsk, about $430 \mathrm{~m}$ a.s.l. $\left(52.49^{\circ} \mathrm{N} 103.91^{\circ} \mathrm{E}\right)$; Bolshoje Goloustnoje enviroment, about $500 \mathrm{~m}$ a.s.l. $\left(52.06^{\circ} \mathrm{N} 105.42^{\circ} \mathrm{E}\right)$; Irkutsk, about $440 \mathrm{~m}$ a.s.l. (52.28 $\left.{ }^{\circ} \mathrm{N} 104.31^{\circ} \mathrm{E}\right)$; Sarma enviroment, $600-930 \mathrm{~m}$ a.s.l. $\left(53.10^{\circ} \mathrm{N} 106.84^{\circ} \mathrm{E}\right)$; UstOrdynski east, wetland near steppe, $554 \mathrm{~m}$ a.s.l. $\left(52.84^{\circ} \mathrm{N}\right.$ $\left.104.79^{\circ} \mathrm{E}\right)$.

Eastern Siberia, Buryat Republic (Fig. 1 Map 1). EastSajan, Mondy, $30 \mathrm{~km}$ northwest, Camp near the Black Irkut, $1878 \mathrm{~m}$ a.s.l. $\left(51.91^{\circ} \mathrm{N} 100.76^{\circ} \mathrm{E}\right)$; Bank of Black Irkut, $1691 \mathrm{~m}$ a.s.l. $\left(51.80^{\circ} \mathrm{N} 100.72^{\circ} \mathrm{E}\right)$.
Eastern Siberia, Zabaikalskii Territory (Fig. 1, Map 1). Daurskij Reserve, $650 \mathrm{~m}$ a.s.l. $\left(49.96^{\circ} \mathrm{N} 115.43^{\circ} \mathrm{E}\right)$.

Far East, Khabarovsk Territory (Fig. 1, Map 2). Boitsovo $\left(47.08^{\circ} \mathrm{N} 134.30^{\circ} \mathrm{E}\right)$.

Far East, Primorskii Territory (Fig. 1, Map 2). Anisimovka, $70 \mathrm{~km}$ east Vladivostok, $240-480 \mathrm{~m}$ a.s.l. $\left(43.18^{\circ} \mathrm{N}\right.$ $\left.132.76^{\circ} \mathrm{E}\right)$; Bonevurovo $\left(43.72^{\circ} \mathrm{N} 132.04^{\circ} \mathrm{E}\right)$; Chasan $7 \mathrm{~km}$ west, about $20 \mathrm{~m}$ a.s.1. $\left(42.43^{\circ} \mathrm{N} 130.65^{\circ} \mathrm{E}\right)$; Dalnegorsk northwest, $719 \mathrm{~m}$ a.s.l. $\left(44.64^{\circ} \mathrm{N} 135.47^{\circ} \mathrm{E}\right)$; Kavalerovo, $200 \mathrm{~m}$ a.s.l. $\left(44.25^{\circ} \mathrm{N} 135.06^{\circ} \mathrm{E}\right)$; Lazo $\left(43.38^{\circ} \mathrm{N} 133.90^{\circ} \mathrm{E}\right)$; Mezhdurechje $\left(45.75^{\circ} \mathrm{N} 134.12^{\circ} \mathrm{E}\right)$; Novonezhino southeast, $138 \mathrm{~m}$ a.s.l. $\left(43.19^{\circ} \mathrm{N} 132.66^{\circ} \mathrm{E}\right)$; Poshiga $2 \mathrm{~km}$ south Ariadnoje, $233-270 \mathrm{~m}$ a.s.l. $\left(45.12^{\circ} \mathrm{N} 134.38^{\circ} \mathrm{E}\right)$; Ryazanovka $\left(42.79^{\circ} \mathrm{N} 131.24^{\circ} \mathrm{E}\right)$; Samarka, Gordeyevska Mt. $\left(44.74^{\circ} \mathrm{N} 134.21^{\circ} \mathrm{E}\right)$; Saratovka $8 \mathrm{~km}$ south, $173 \mathrm{~m}$ a.s.l. (44.58 $\left.{ }^{\circ} \mathrm{N} 134.13^{\circ} \mathrm{E}\right)$; Kalinovka, $171-259 \mathrm{~m}$ a.s.l. $\left(44.45^{\circ} \mathrm{N}\right.$ $\left.132.95^{\circ} \mathrm{E}\right)$; Tikhoye nr. Razdolnoye, $36 \mathrm{~km}$ south Ussuriysk $\left(43.60^{\circ} \mathrm{N} 131.87^{\circ} \mathrm{E}\right)$; Uborka north, $206 \mathrm{~m}$ a.s.l. $\left(44.45^{\circ} \mathrm{N} 134.07^{\circ} \mathrm{E}\right)$.

Far East, Sakhalin (Fig. 1 Map 2). Yuzhno-Sakhalinsk, about $50 \mathrm{~m}$ a.s.l. $\left(46.96^{\circ} \mathrm{N} 142.73^{\circ} \mathrm{E}\right)$; Korsakov, Ptitche Lake, about $50 \mathrm{~m}$ a.s.l. $\left(46.63^{\circ} \mathrm{N} 142.79^{\circ} \mathrm{E}\right)$; Lugovoje, about $50 \mathrm{~m}$ a.s.l. $\left(47.03^{\circ} \mathrm{N} 142.79^{\circ} \mathrm{E}\right)$; Novoaleksandrovsk, about $50 \mathrm{~m}$ a.s.l. $\left(47.05^{\circ} \mathrm{N} 142.73^{\circ} \mathrm{E}\right)$; Pravda, about $50 \mathrm{~m}$ a.s.l. $\left(46.94^{\circ} \mathrm{N} 142.01^{\circ} \mathrm{E}\right)$.

\section{List of species}

\section{Sphecidae}

\section{Ammophila deserticola TSUNEKI, 1971}

Irkutsk Province. 2 o $^{\star}$ o $^{\star}$ Bolshoje Goloustnoje 20.06.2011, leg. Liebig.

Distribution: Russia: Urals, Western and Eastern Siberia, Far East. - Kyrgyzstan, Mongolia, China.

\section{Ammophila infesta F. SMIтH, 1873}

Primorskii Territory. $1 \sigma^{\star}$ Anisimovka 01.07.2014, $1 \sigma^{\star}$, 1 ㅇ Poshiga 21.06.2014, leg. Liebig.

Distribution: Russia: Far East. - Korean Peninsula, Japan.

\section{Ammophila mongolensis TSUNEKI, 1971 \\ (Fig. 2a)}

Buryat Republic. 1 ơ, 3 ㅇ ㅇ East Sajan, Mondy, $30 \mathrm{~km}$ NW 07.-11.07.2012, leg. Liebig.

Distribution: Russia: Western and Eastern Siberia. Turkey, Tajikistan, Uzbekistan, Kyrgyzstan, Mongolia, China. 


\section{Ammophila pubescens CURTIS, 1836}

Irkutsk Province. $12 o^{x}, 6$ 우 Bolshoje Goloustnoje 11.-24.06.2011, leg. Liebig, $1 \sigma^{\star}$ Bolshoje Goloustnoje 18.07.2012, leg. Floßmann. $80^{\star} o^{\star}, 6$ 우 ㅇ Sarma 16.-22. 07.2007, leg. Liebig.

Buryat Republic. $8 \sigma^{\star} \sigma^{*}, 1$ ㅇ East Sajan, Mondy, $30 \mathrm{~km}$ NW 29.06.-13.07.2012, leg. Liebig.

Primorskii Territory. $1 \sigma^{\star}$ Poshiga 21.06.2014, leg. Liebig.

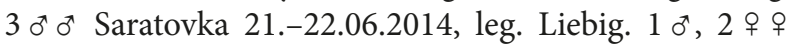
Kalinovka 26.-27.06.2014, leg. Liebig.

Distribution: Russia: European Part, Urals, Western and Eastern Siberia, Far East. - Europe, Turkey, Iran, Kazakhstan, Mongolia, China.

\section{Ammophila sabulosa (LinNé, 1758)}

Irkutsk Province. $4 o^{x} o^{x}, 1$ ㅇ Bolshoje Goloustnoje 12.-22.06.2011, leg. Liebig, 1 i Bolshoje Goloustnoje 18.07.2012, leg. Floßmann. 2 o o o $^{\star}$ Sarma 19./24.07.2007, leg. Liebig.

Distribution: Russia: European Part, Urals, Western and Eastern Siberia, Far East. - Europe, North Africa, Turkey, Syria, Iran, Central Asia, Kazakhstan, Mongolia, China.

\section{Ammophila sickmanni KoHL, 1901}

Primorskii Territory. 2 ๙ $\sigma^{\text {๙ }}$, 1 ㅇ Kalinovka 26.-27.06. 2014, leg. Liebig. 1 o Poshiga 20.06.2014, leg. Liebig. 1 ㅇ Anisimovka 02.07.2014, leg. Liebig.

Distribution: Russia: Eastern Siberia, Far East. Mongolia, China, Korean Peninsula.

\section{Ammophila terminata F. SMiтH, 1856}

Buryat Republic. 1 o $^{\star}$ East Sajan, Mondy, $30 \mathrm{~km} \mathrm{NW}$ 13.07.2012, leg. Liebig.

Distribution: Russia: European Part, Urals, Western and Eastern Siberia. - Europe, North Africa, Turkey, Central Asia, Kazakhstan, Mongolia, China.

\section{Ammophila vagabunda F. SMITH, 1856}

Primorskii Territory. $1 \sigma^{*}$ Anisimovka 02.07.2014, leg. Liebig. 1 क Anisimovka 07.06.1993, leg. Taeger. $10^{\text {t }}$ Novonezhino 30.06.2014, leg. Liebig. 1 o $^{\star}$ Poshiga 21. 06.2014, leg. Liebig.

Distribution: Russia: Far East. - Mongolia, China, Korean Peninsula, Japan, Southeast Asia, India.

\section{Chalybion japonicum (GRIBOD0, 1883)}

Primorskii Territory. $2 \sigma^{\star} \sigma^{\star}, 3$ ㅇ f Kalinovka 29.06.2014, leg. Liebig.

Distribution: Russia: Far East. - China, Korean Peninsula, Japan.

\section{Hoplammophila aemulans (KoHL, 1901)}

Primorskii Territory. $10^{\star}, 2$ 우 우 Kalinovka 29.06.2014, leg. Liebig.

Distribution: Russia: Far East. - China, Korean Peninsula, Japan.

\section{Palmodes occitanicus (LePELETIeR \& SeRVILLE, 1828)}

Irkutsk Province. $10 o^{\star} o^{\star}, 2$ 우 우 Bolshoje Goloustnoje 12.-26.06.2011, leg. Liebig, partim det. Schmid-Egger.

Distribution: Russia: European Part, Western and Eastern Siberia, Far East. - Europe, North Africa, Turkey, Syria, Israel, Iran, Central Asia, Kazakhstan, Mongolia, China, Korean Peninsula.

Remark: In the opinion of Schmid-EgGer (personal communication) the specimens from the Far East may represent a separate species. A revision of the Palaearctic species of Palmodes KoHL, 1890 is outstanding.

\section{Podalonia affinis (KIRBY, 1798)}

Irkutsk Province. $3 \sigma^{\star} o^{x}, 5$ 우 Bolshoje Goloustnoje 12.-20.06.2011, leg. Liebig.

Distribution: Russia: European Part, Urals, Western and Eastern Siberia, Far East. - Europe, North Africa, Caucasus, Turkey, Jordan, Israel, Iran, Central Asia, Kazakhstan, Mongolia, China, Korean Peninsula.

\section{Podalonia alpina (KOHL, 1888)}

${ }^{\star}$ Buryat Republic. 5 o $^{\star} \mathrm{o}^{\star}, 4$ 우 우 Mondy, $30 \mathrm{~km} \mathrm{NW}$ 28.06.-13.07.2014, leg. Liebig.

Distribution: Russia: European Part (North Caucasus), Western Siberia (Altai), Eastern Siberia (Tuva Republic). - Mountains of Europe, North Africa, Turkey, Iran, Afghanistan, Tajikistan, Kyrgyzstan, Kazakhstan, Mongolia, China.

\section{Podalonia asiatica DANILov, 2017}

${ }^{\star}$ Irkutsk Province. $10 \sigma^{\star} \sigma^{\star}$ Sarma 17.-24.07.2007, leg. Liebig.

Distribution: Russia: Eastern Siberia. - Mongolia, China. 
Remark: The present specimens are running in the keys of KoHl (1906) and Dollfuss (2010) to Podalonia andrei (F. Morawitz, 1889). After revision of the group by DANILOv (2017) the males are conspecific with Podalonia asiatica. Maybe two 우 ㅇ from the same place belongs to the hitherto unknown females of this species.

\section{Podalonia caucasica (Mocsáry, 1883)}

Irkutsk Province. 1 \& Sarma 16.07.2007, leg. Liebig det. Schmid-Egger. 7 o $^{\star \top}, 20$ 우 우 Bolshoje Goloustnoje 12.-25.06.2011, leg. Liebig.

Buryat Republic. East Sajan, Mondy, $30 \mathrm{~km}$ NW 1 ㅇ 25.06.2012, leg. Liebig.

Distribution: Russia: Western and Eastern Siberia.- Bulgaria (Dollfuss 2010), Georgia, Tajikistan, Uzbekistan, Kazakhstan, Mongolia, China, India. The record Estonia (Europe EE) (Belokobylskij \& LeLEJ 2017) may be an error.

\section{Sceliphron deforme (F. SMIтH, 1856)}

Primorskii Territory. 2 ㅇ ㅇ Novonezhino 01.07.2014, leg. Liebig det. Schmid-Egger. 3 o $\sigma^{\top}$ Kalinovka 29.06.2014, leg. Liebig, partim det. Schmid-Egger.

Distribution: Russia: European Part, Western and Eastern Siberia, Far East. - Europe, Tajikistan, Uzbekistan, Kyrgyzstan, Kazakhstan, Mongolia, China, Korean Peninsula, Japan, Southeast Asia, India.

\section{Sphex funerarius GuSSAKOvSKIJ, 1934}

Irkutsk Province. 1 o $^{\star}$ Bolshoje Goloustnoje 21.06.2011, leg. Liebig, det. Schmid-Egger.

Distribution: Russia: European Part, Urals, Western and Eastern Siberia, Far East. - Europe, North Africa, Georgia, Turkey, Syria, Israel, Iran, Central Asia, Kazakhstan, Mongolia, China.

\section{Crabronidae}

\section{Alysson pertheesi GORSKI, 1852}

Irkutsk Province. 1 ㅇ Sarma 24.07.2007, leg. Liebig.

Distribution: Russia: European Part, Western and Eastern Siberia, Far East. - Europe, Mongolia, China, Korean Peninsula, Japan.

\section{Argogorytes fargeii (SHUCKARD, 1837)}

Irkutsk Province. $2 \sigma^{\star} o^{\star}, 8$ 우 Bolshoje Goloustnoje 19.-25.06.2011, leg. Liebig, partim det. Schmid-Egger.
Distribution: Russia: European Part, Urals, Western and Eastern Siberia. - Europe, Armenia, Turkey, Israel, Kazakhstan, Mongolia, China.

\section{Argogorytes mystaceus grandis (GUSSAKOvSKIJ, 1932)}

Primorskii Territory. $1 \sigma^{\top}$ Anisimovka 07.06.1993, leg. Taeger. $10^{\star}$ Anisimovka 07.06.1993, leg. Taeger. 1 ㅇ Poshiga 20.06.2014, leg. Liebig, det. Schmid-Egger. 1 ㅇ Kalinovka 29.06.2014, leg. Liebig, det. Schmid-Egger.

Distribution: Russia: Eastern Siberia, Far East. - China, Korean Peninsula, Japan.

Remark: In opinion of ScHMID-EgGer (personal communication), A. grandis is a distinct species.

\section{Astata boops (SCHRANK, 1781)}

Irkutsk Province. 1 o Bolshoje Goloustnoje 20.06.2011, leg. Liebig. $1 \sigma^{\text {त }}$ Ust-Ordynski (steppe) 27.06.2011, leg. Liebig.

Distribution: Russia: European Part, Urals, Western and Eastern Siberia, Far East. - Europe, North Africa, Abkhazia, Turkey, Israel, Arabian Peninsula, Iraq, Iran, Afghanistan, Turkmenistan, Tajikistan, Uzbekistan, Kazakhstan, Mongolia, China, Korean Peninsula, India.

\section{Astata minor KoHL, 1885}

Irkutsk Province. $1 \sigma^{\star}$ Bolshoje Goloustnoje 25.06.2011, leg. Liebig. $2 \sigma^{\top} o^{\star}, 1+$ Ust-Ordynski (Steppe) 26.27.06.2011, leg. Liebig.

Distribution: Russia: European Part, Western and Eastern Siberia, Far East. - Europe, North Africa, Turkey, Israel, Iran, Turkmenistan, Uzbekistan, Kazakhstan.

\section{Bembix diversipes F. Morawitz, 1889}

Irkutsk Province. $10 \sigma^{\star} \sigma^{\star}, 6$ 우 + Sarma 16.-22.07.2007, leg. Liebig, partim det. Schmid-Egger. $9 \sigma^{\top} \sigma^{\star \top}$ Bolshoje Goloustnoje 20.-25.06.2011, leg. Liebig. 3 क $0^{\star}, 4$ 우 우 UstOrdynski 26.-27.06.2011, leg. Liebig.

Distribution: Russia: Western and Eastern Siberia, Far East. - Turkey, Iran, Uzbekistan, Kyrgyzstan, Kazakhstan, Mongolia, China.

\section{Bembix rostrata rostrata (LINNÉ, 1758)}

Irkutsk Province. $9 \sigma^{\star} \sigma^{\star}, 4$ 우 S Sarma 24.07.2007, leg. Liebig, partim det. Schmid-Egger. $3 \sigma^{\star} \sigma^{\star}$ Ust-Ordynski 26.06.2011, leg. Liebig. 
Distribution: Russia: European Part, Urals, Western and Eastern Siberia. - Europe, Azerbaijan, Turkey, Iran, Turkmenistan, Tajikistan, Uzbekistan, Kazakhstan, Mongolia, China.

\section{Cerceris arenaria (LInNÉ, 1758)}

Irkutsk Province. $1 \sigma^{\star}$ Bolshoje Goloustnoje 22.06.2011, leg. Liebig. $14 o^{\star} o^{\star}, 14$ 우 우 Sarma 17.-24.07.2007, leg. Liebig.

Distribution: Russia: European Part, Urals, Western and Eastern Siberia, Far East. - Europe, North Africa, Caucasus, Turkey, Israel, Iran, Central Asia, Kazakhstan, Mongolia, China, Korean Peninsula, Japan.

Remark: According to SCHMIDT (2000) Cerceris arenaria stecki SCHLETTERER, 1889 differs from the nominate form only in more whitish and smaller bands on the tergites. Nemkov (2009) did not treat it as a separate taxon. We agree with his opinion.

\section{Cerceris hortivaga KoHL, 1880}

Primorskii Territory. 2 우 우 Anisimovka 02.07.2014, leg. Liebig. 1 † Kalinovka 28.06.2014, leg. Liebig.

Distribution: Russia: Far East. - Europe, China, Korean Peninsula, Japan.

\section{Cerceris micropunctata SHESTAKOV, 1918}

Irkutsk Province. $3 \sigma^{\star} o^{\star}, 1$ ㅇ Bolshoje Goloustnoje 13.-20.06.2011, leg. Liebig. 3 i ㅇ Sarma 18.07.2007, leg. Liebig.

Distribution: Russia: Western and Eastern Siberia.

\section{Cerceris pedetes KOHL, 1887}

*Irkutsk Province. 2 ㅇ ㅇ Sarma 24.07.2007, leg. Liebig.

Distribution: Russia: Eastern Siberia, Far East. - China, Korean Peninsula, Japan.

\section{Cerceris quadrifasciata (PANZER, 1799)}

Irkutsk Province. 1 i Bolshoje Goloustnoje 25.06.2011, leg. Liebig. 1 क Sarma 23.07.2007, leg. Liebig.

Distribution: Russia: European Part, Urals, Western and Eastern Siberia, Far East. - Europe, Caucasus, Turkey, Kazakhstan, China, Korean Peninsula.

\section{Cerceris ruficornis (FABRICIUS, 1793)}

Irkutsk Province. $4 \sigma^{*} \sigma^{*}$ Bolshoje Goloustnoje 24.-25.

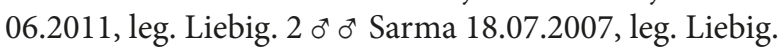

Distribution: Russia: European Part, Urals, Western and Eastern Siberia, Far East. - Europe, North Africa, Turkey, Israel, Iran, Tajikistan, Uzbekistan, Kyrgyzstan, Kazakhstan, China, Korean Peninsula.

\section{Cerceris rybyensis (LINNÉ, 1771)}

Irkutsk Province. $1 \sigma^{\pi}, 1$ ㅇ Bolshoje Goloustnoje 25.06.2011, leg. Liebig.

Distribution: Russia: European Part, Urals, Western and Eastern Siberia, Far East. - Europe, North Africa, Caucasus, Turkey, Syria, Iran, Turkmenistan, Tajikistan, Uzbekistan, Kazakhstan, Korean Peninsula, Japan, India.

\section{Crabro (Anothyreus) lapponicus ZetTERSTEDT, 1838}

(Fig. 2b)

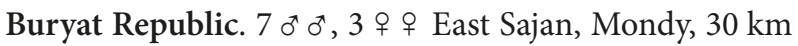
NW 28.06.-13.07.2012, leg. Liebig.

Distribution: Russia: European Part (north and northwest), Eastern Siberia. - Europe (northern parts).

\section{Crabro (Anothyreus) maeklini A. MorawITz, 1866 \\ (Figs. 2c, 2d)}

${ }^{\star}$ Buryat Republic. $13 o^{\star} o^{\star}, 7$ i $~+$ East Sajan, Mondy, 30 km NW 28.06.-14.07.2012, leg. Liebig.

Distribution: Russia: European Part, Urals, Eastern Siberia, Far East. - Europe (northern parts), Kazakhstan, Mongolia.

\section{Crabro (Crabro) cribrarius (LinNÉ, 1758)}

Irkutsk Province. $3 \sigma^{x} o^{x}, 2$ 우 우 Bolshoje Goloustnoje 20.-22.06.2011, leg. Liebig.

Zabaikalskii Territory. $1{ }^{\star}$ Daurskij Reserve 24.07.1989, leg. Nesterov.

Sakhalin. 1 o Korsakov (Ptitche Lake) 12.08.1975, leg. Nesterov.

Distribution: Russia: European Part, Urals, Western and Eastern Siberia, Far East. - Europe, Turkey, Iran, Kazakhstan, Mongolia, Korean Peninsula. The record from North Africa by BeLoKobylskij \& Lelej (2017) is doubtful.

\section{Crabro (Crabro) peltarius (SCHREBER, 1784)}

Sakhalin. 1 o Novoaleksandrovsk [without date], leg. Nesterov. 
Distribution: Russia: European Part, Urals, Western and Eastern Siberia, Far East. - Europe, Turkey, Kazakhstan, Mongolia, Korean Peninsula.

\section{Crabro (Crabro) sibiricus A. MoRawitz, 1866}

Zabaikalskii Territory. 1 o Daurskij Reserve 24.07.1989, leg. Golovushkin.

Distribution: Russia: Western and Eastern Siberia, Far East. - Kazakhstan, Mongolia, China.

\section{Crossocerus (Acanthocrabro) vagabundus (PANZER, 1798)}

Irkutsk Province. 2 우 우 Bolshoje Goloustnoje 20./24. 06.2011, leg. Liebig. 1 đo Sarma 18.07.2007, leg. Liebig.

Distribution: Russia: European Part, Urals, Western and Eastern Siberia, Far East. - Europe, North Africa, Turkey, Uzbekistan, Kazakhstan, Mongolia, China, Korean Peninsula, Japan, India.

\section{Crossocerus (Blepharipus) barbipes (DAHLBOM, 1845)}

Irkutsk Province. 1 ㅇ Sarma 19.07.2007, leg. Liebig.

Distribution: Russia: European Part, Urals, Western and Eastern Siberia, Far East. - Europe, Turkey, Kazakhstan, Mongolia, China, Korean Peninsula, Japan, North America.

\section{Crossocerus (Blepharipus) cetratus (SHucKARD, 1837)}

Irkutsk Province. 1 ㅇ Sarma 16.07.2007, leg. Liebig. 1 ㅇ Bolshoje Goloustnoje 13.06.2011, leg. Liebig.

Distribution: Russia: European Part, Urals, Western and Eastern Siberia, Far East. - Europe, Turkey, Kazakhstan, Mongolia, China, Korean Peninsula, Japan.

\section{Crossocerus (Blepharipus) leucostoma (LINNÉ, 1758)}

${ }^{\star}$ Buryat Republic. 1 ㅇ East Sajan, Mondy, $30 \mathrm{~km}$ NW 30.06.2012, leg. Liebig.

Primorskii Territory. 1 o $^{\star}$ Anisimovka 07.06.1993, leg. Taeger. 1 ㅇ Dalnegorsk 24.06.2014, leg. Liebig.

Distribution: Russia: European Part, Urals, Western and Eastern Siberia, Far East. - Europe, Turkey, Kazakhstan, Mongolia, Korean Peninsula, Japan, North America.

\section{Crossocerus (Blepharipus) walkeri (SHuckARD, 1837)}

Irkutsk Province. $1 \sigma^{\star}, 1$ \& Sarma 18./19.07.2007, leg. Liebig.
Distribution: Russia: European Part, Urals, Western and Eastern Siberia, Far East. - Europe, Turkey, Kazakhstan, Mongolia, Korean Peninsula, Japan.

\section{Crossocerus (Crossocerus) elongatulus (VANDER LINDEN, 1829)}

Irkutsk Province. 1 o $^{\text {t }}$ Angarsk 02.07.1983, leg. Nemkov. ${ }^{*}$ Buryat Republic. 1 i East Sajan, Mondy, $30 \mathrm{~km} \mathrm{NW}$ 02.07.2012, leg. Liebig.

Distribution: Russia: European Part, Urals, Western and Eastern Siberia, Far East. - Europe, North Africa, Azerbaijan, Turkey, Iran, Turkmenistan, Tajikistan, Turkmenistan, Kyrgyzstan, Kazakhstan, Mongolia, Japan, North and South America.

Remark: The differentiation between the subspecies C. elongatulus (s.str.) and C.elongatulus annulatus LEPELETIER \& BRULlé, 1835 as made by BeLOKOBYLSKIJ \& LELEJ (2017) seems doubtful. The common occurrence of two subspecies in a wide range of distribution contradicts the concept of subspecies. Here is a need of clearing the taxonomy and the distribution data. So we don't follow this opinion.

\section{Crossocerus (Crossocerus) exiguus (VANDER Linden, 1829)}

Irkutsk Province. 1 ㅇ Sarma 21.07.2007, leg. Liebig.

Distribution: Russia: European Part, Urals, Western and Eastern Siberia, Far East. - Europe, North Africa, Turkmenistan, Kazakhstan, Mongolia, China, Korean Peninsula.

\section{Crossocerus (Crossocerus) kohli (BIschoff, 1921)}

${ }^{\star}$ Buryat Republic. 2 우 우 East Sajan, Mondy, $30 \mathrm{~km}$ NW 11.-12.07.2012, leg. Liebig.

Distribution: Russia: Western and Eastern Siberia, Far East. - Tajikistan, Uzbekistan, Kyrgyzstan, Kazakhstan, Mongolia, China, India.

\section{Crossocerus (Crossocerus) palmipes palmipes (LINNÉ, 1767)}

Irkutsk Province. 1 \& Sarma 24.07.2007, leg. Liebig.

Distribution: Russia: European Part, Urals, Western and Eastern Siberia. - Europe, North Africa, Turkey, Kazakhstan, Mongolia.

\section{Crossocerus (Crossocerus) tarsatus (SHuckARD, 1837)}

${ }^{\star}$ Buryat Republic. $1 \sigma^{\star}$ East Sajan, Mondy, $30 \mathrm{~km} \mathrm{NW}$ 13.07.2012, leg. Liebig. 
Distribution: Russia: European Part, Western Siberia, Far East. - Europe, North Africa, Georgia, Turkey, Iran, Kazakhstan, Mongolia, Korean Peninsula, North America. New record for Eastern Siberia.

\section{Crossocerus (Crossocerus) yasumatsui (TSUNEKI, 1947)}

*Tuva Republic. 8 ơ ơ Kysyl 16.08.1998, leg. Mattes. Buryat Republic. $15 \sigma^{\star} o^{\star}, 4$ 우 아 East Sajan, Mondy, $30 \mathrm{~km}$ NW 10.-13.07.2012, leg. Liebig.

Distribution: Russia: Western and Eastern Siberia, Far East. - Kyrgyzstan, Kazakhstan, Mongolia, Japan, South East Asia.

\section{Crossocerus (Cuphopterus) dimidiatus (FABRICIUS, 1781)}

Irkutsk Province. 3 đ $\sigma^{\star}$ Bolshoje Goloustnoje 19.06. 2011, leg. Liebig.

Sakhalin. $7 \sigma^{\top} \sigma^{\top}$ Lugovoje 22.07.1980, leg. Nesterov. $8 \sigma^{\star \top} \sigma^{\star}$ Pravda 28.08.1995, leg. Nesterov.

Distribution: Russia: European Part, Urals, Western and Eastern Siberia, Far East. - Europe, North Africa, Turkey, Kyrgyzstan, Kazakhstan, Mongolia, China, Korean Peninsula, Japan.

Remark: C. dimidiatus (s. str.) occurs in the Far East and the western parts of Siberia, C. dimidiatus agyo Terayama \& Takahashi, 2017 on Sakhalin, the Kuriles, Korean Peninsula and Japan. Nemкov et al. (1995) made no difference between both subspecies. The latter was described by КонL (1915) as Crossocerus serripes sapporoensis after a single female with darker legs. The male differs from the nominate form by less yellow markings (see LECLERCQ 1974). Maybe these are color variants only.

\section{Crossocerus (Hoplocrabro) quadrimaculatus (FABRICIUS, 1793)}

Irkutsk Province. 2 ㅇ ㅇ Sarma 17./18.07.2007, leg. Liebig.

Distribution: Russia: European Part, Urals, Western and Eastern Siberia, Far East. - Europe, North Africa, Turkey, Iran, Tajikistan, Uzbekistan, Kazakhstan, Mongolia.

\section{Diodontus collaris TSUNEKI, 1972}

Irkutsk Province. $6 o^{\star} o^{\star}$ Sarma 18.-24.07.2007, leg. Liebig. ${ }^{\star}$ Buryat Republic. $12 \sigma^{\star} o^{\star}$, 5 ㅇ East Sajan, Mondy, 30 km NW 06.-13.07.2012, leg. Liebig.

Distribution: Russia: Western and Eastern Siberia. Kazakhstan, Mongolia.

\section{Diodontus dziuroo TSUNEKI, 1972}

${ }^{\star}$ Irkutsk Province. 1 ㅇ Bolshoje Goloustnoje 20.06.2011, leg. Liebig.

Distribution: Russia: Eastern Siberia, Far East. - Kazakhstan, Mongolia.

\section{Diodontus gegen TSUNEKI, 1972}

${ }^{\star}$ Irkutsk Province. 1 đ Bolshoje Goloustnoje 20.06.2011, leg. Liebig.

Distribution: Russia: Eastern Siberia. - Mongolia.

\section{Diodontus handlirschi KoHL, 1888}

${ }^{\star}$ Buryat Republic. $1 \sigma^{\star}$ East Sajan, Mondy, $30 \mathrm{~km} \mathrm{NW}$ 01.07.2012, leg. Liebig.

Distribution: Russia: Western Siberia (Altai), Far East. Europe, North Africa, Turkey, Kyrgyzstan, Kazakhstan, Mongolia. New record for Eastern Siberia.

\section{Diodontus luperus SHUCKARD, 1837}

Irkutsk Province. 1 \& Bolshoje Goloustnoje 22.06.2011, leg. Liebig.

Distribution: Russia: European Part, Urals, Western and Eastern Siberia. - Europe, North Africa, Turkey, Kazakhstan, Mongolia.

\section{Diodontus medius DAHLBOM, 1844}

Irkutsk Province. $2 \sigma^{\star} o^{\star}$ Bolshoje Goloustnoje 16.06. 2011. 1 đ`, 8 우 ㅇ Sarma 16.-24.07.2007, leg. Liebig. *Buryat Republic. 4 o $^{\star} o^{\star}$ East Sajan, Mondy, $30 \mathrm{~km} \mathrm{NW}$ 30.06.2012, leg. Liebig.

Distribution: Russia: European Part, Urals, Western and Eastern Siberia, Far East. - Europe, Turkey.

\section{Diodontus tristis (VANDER LINDEN, 1829)}

Irkutsk Province. $1 \sigma^{\top}$ Bolshoje Goloustnoje 16.06.2011, leg. Liebig. $1 o^{x}, 1$ ㅇ Sarma 21./23.07.2007, leg. Liebig.

Distribution: Russia: European Part, Urals, Western and Eastern Siberia, Far East. - Europe, Turkey, Iran, Tajikistan, Uzbekistan, Kyrgyzstan, Kazakhstan, Mongolia.

\section{Dryudella pinguis (DAHLBOM, 1832)}

Irkutsk Province. $1 \sigma^{\star}, 3$ 우 Bolshoje Goloustnoje 11.-24.06.2011, leg. Liebig. 1 o , 1 क Sarma 17./18.07.2007, leg. Liebig. 1 o Ust-Ordynski (steppe) 27.06.2011, leg. Liebig. 
${ }^{\star}$ Buryat Republic. 2 우 우 East-Sajan, Mondy, 30 km NW 06.07.2012, leg. Liebig.

Distribution: Russia: European Part, Western and Eastern Siberia, Far East. - Europe, Kazakhstan, North America.

\section{Dryudella stigma (PANZER, 1809)}

Irkutsk Province. $2 o^{\star} o^{\star}, 4$ 우 우 Sarma 16.-24.07.2007, leg. Liebig.

Distribution: Russia: European Part, Western and Eastern Siberia, Far East. - Europe, Kazakhstan.

\section{Ectemnius (Clytochrysus) cavifrons (Thomson, 1870)}

Irkutsk Province. 1 o, 1 우 Bolshoje Goloustnoje 13./23. 06.2011, leg. Liebig.

Primorskii Territory. $3 \sigma^{\star} \sigma^{\star}$ Poshiga 20./21.06.2014, leg. Liebig.

Sakhalin. $2 \sigma^{*} \sigma^{*}, 1$ ㅇ Korsakov (Ptitche Lake) 12.08.1975, leg. Nesterov. $5 \sigma^{\top} \sigma^{\star}$ Lugovoje 22.07.1980, leg. Nesterov. $2 o^{\top} o^{x}, 1$ i Novoaleksandrovsk (without date), leg. Nesterov.

Distribution: Russia: European Part, Urals, Western and Eastern Siberia, Far East. - Europe, North Africa, Tajikistan, Kazakhstan, Mongolia, Korean Peninsula, Japan, South East Asia.

\section{Ectemnius (Clytochrysus) lapidarius (PAnzeR, 1803)}

Primorskii Territory. 1 \& Bonevurovo 25.05.1993, leg. Taeger. 1 o Ryazanovka 16.06.1993, leg. Taeger. Sakhalin. $10^{*}$ Korsakov (Ptitche Lake) 12.08.1975, leg. Nesterov. 1 ㅇ Lugovoje 22.07.1980, leg. Nesterov.

Distribution: Russia: European Part, Urals, Western and Eastern Siberia, Far East. - Europe, North Africa, Georgia, Turkey, Iran, Kyrgyzstan, Kazakhstan, Mongolia, China, Korean Peninsula, Japan, North America.

\section{Ectemnius (Clytochrysus) ruficornis (ZETTERSTEDT, 1838)}

Irkutsk Province. 1 o Bolshoje Goloustnoje 14.06.2011, leg. Liebig.

Sakhalin. $2 \sigma^{*} \sigma^{*}$ Korsakov (Ptitche Lake) 12.08.1975, leg. Nesterov. $2 \sigma^{*} \sigma^{*}$ Lugovoje 22.07.1980, leg. Nesterov. $2 \sigma^{*} \sigma^{*}$ Novoaleksandrovsk (without date), leg. Nesterov. $10^{\circ}$ Pravda 28.08.1995, leg. Nesterov.

Distribution: Russia: European Part, Urals, Western and Eastern Siberia, Far East. - Europe, Turkey, Kazakhstan, Mongolia, Korean Peninsula, Japan, South East Asia, North America.

\section{Ectemnius (Clytochrysus) sexcinctus (FABRICIUs, 1775)}

^Irkutsk Province. 1 o Sarma 21.07.2007, leg. Liebig.

Distribution: Russia: European Part, Western and Eastern Siberia, Far East. - Europe, North Africa, Turkey, Iran, Kyrgyzstan, Kazakhstan, Mongolia, China, India, North America.

\section{Ectemnius (Ectemnius) borealis (ZetTerstedT, 1838)}

Irkutsk Province. $3 \sigma^{\star} \sigma^{\star}, 1$ ㅇ Bolshoje Goloustnoje 19.-25.06.2011, leg. Liebig. $3 o^{\top} o^{\star}, 6$ 우 우 Sarma 16.-19. 07.2007, leg. Liebig.

Zabaikalskii Territory.1 क Daurskij Reserve 24.07.1989, leg. Nesterov.

Primorskii Territory. 1 ㅇ Poshiga 21.06.2014, leg. Liebig.

Sakhalin. 1 i Lugovoje 22.07.1980, leg. Nesterov. 1 ㅇ Novoaleksandrovsk (without date), leg. Nesterov.

Distribution: Russia: European Part, Urals, Western and Eastern Siberia, Far East. - Europe, Turkey, Kazakhstan, Mongolia, Korean Peninsula, Japan, North America.

\section{Ectemnius (Ectemnius) dives (Lepeletier \& BRullé,} 1835)

Primorskii Territory. $1 \sigma^{\star}$ Anisimovka 07.06.1993, leg. Taeger.

Sakhalin. $1 \sigma^{\top}$ Lugovoje 22.07.1980, leg. Nesterov.

Distribution: Russia: European Part, Urals, Western and Eastern Siberia, Far East. - Europe, North Africa, Georgia, Turkey, Israel, Kazakhstan, China, Korean Peninsula, Japan, North America.

\section{Ectemnius (Ectemnius) guttatus (VANDER LINDEN 1829)}

Irkutsk Province. $2 \sigma^{\star} o^{*}$ Bolshoje Goloustnoje 14./ 24.06.2011, leg. Liebig. $10^{\star}$ Bolshoje Goloustnoje 18.06.2011, leg. Jänicke.

*Sakhalin: 10 Korsakov (Ptitche Lake) 12.08.1975, leg. Nesterov. $2 \sigma^{\star} o^{\star}, 1$ ㅇ Lugovoje 22.07.1980, leg. Nesterov. 1 o Pravda 28.08.1995, leg. Nesterov.

Distribution: Russia: European Part, Urals, Western and Eastern Siberia. - Europe, Kazakhstan. New record for Far East.

\section{Ectemnius (Hypocrabro) continuus (FABRICIUS, 1804)}

Irkutsk Province. $1 \sigma^{\star}$ Bolshoje Goloustnoje 23.06.2011, leg. Liebig. 1 o Bolshoje Goloustnoje 18.07.2012, leg. Floßmann. 7 o o Sarma 16.-21.07.2007, leg. Liebig. 
Buryat Republic. $10^{\star}, 1$ 우 East-Sajan, Mondy, $30 \mathrm{~km}$ NW 06./12.07.2012, leg. Liebig.

Zabaikalskii Territory. 2 우 우 Daurskij Reserve 24.07.1989, leg. Golovushkin. $10^{\star}$ Daurskij Reserve 24.07.1989, leg. Nesterov.

Primorskii Territory. $1 \sigma^{\star}$ Anisimovka 08.06.1993, leg. Taeger. $1 \sigma^{*}$ Bonevurovo 10.06.1993, leg. Taeger. 1 o Dalnegorsk 25.06.2014, leg. Liebig. 1 o Samarka, Gordeyevska Mt. 29.05.1993, leg. Taeger.

Sakhalin. $70^{\top} o^{\top}$ Yuzhno Sakhalinsk 22.07.1980, leg. Nesterov. 1 \& Korsakov (Ptitche Lake) 12.08.1975, leg. Nesterov. $10^{\star}, 1$ ㅇ Lugovoje 22.07.1980, leg. Nesterov. $10^{\star \top}$ Novoaleksandrovsk (without date), leg. Nesterov.

Distribution: Russia: European Part, Urals, Western and Eastern Siberia, Far East. - Europe, North Africa, Georgia, Azerbaijan, Turkey, Jordan, Israel, Iran, Iraq, Central Asia, Kazakhstan, Mongolia, China, Korean Peninsula, Japan, North and South America.

\section{Ectemnius (Hypocrabro) pedicellaris (F. MoRAwITZ, 1889)}

Zabaikalskii Territory. 1 ㅇ Daurskij Reserve 24.07.1989, leg. Golovushkin.

Distribution: Russia: Eastern Siberia, Far East. - Mongolia, China, Korean Peninsula.

\section{Ectemnius (Hypocrabro) rubicola (Dufour \& PeRRIS, 1840)}

Primorskii Territory. 1 ㅇ Poshiga 20.06.2014, leg. Liebig. Sakhalin. $1 \sigma^{\top}$ Korsakov (Ptitche Lake) 12.08.1975, leg. Nesterov. $30^{\top} o^{\top}$ Lugovoje 22.07.1980, leg. Nesterov. $2 \sigma^{\top} o^{*}$ Pravda 28.08.1995, leg. Nesterov.

Distribution: Russia: European Part, Urals, Western and Eastern Siberia, Far East. - Europe, Turkey, Iran, Kazakhstan, Japan.

\section{Ectemnius (Hypocrabro) schlettereri (KoHL, 1888)}

Irkutsk Province. $1 \sigma^{\star}$ Ust-Ordynski (steppe) 27.06.2011, leg. Liebig.

Primorskii Territory. 1 o $^{\star}$ Poshiga 20.06.2014, leg. Liebig.

Distribution: Russia: European Part, Urals, Western and Eastern Siberia, Far East. - Europe, Iran, Kazakhstan, China, Korean Peninsula, Japan, India, South East Asia.

\section{Ectemnius (Metacrabro) fossorius (LINNÉ, 1758)}

Irkutsk Province. 2 o o $^{\star}$ Sarma 16./18.07.2007, leg. Liebig. Primorskii Territory. 1 ㅇ uborka 23.06.2014, leg. Liebig. 18 ơ o Poshiga 20./21.06.2014, leg. Liebig. 1 i Saratovka 19.06.2014, leg. Liebig.
Sakhalin. $1 \sigma^{*}, 2$ ㅇ ㅇ Lugovoje 22.07.1980, leg. Nesterov. $4 \sigma^{\star} o^{\star}, 1$ \& Novoaleksandrovsk (without date), leg. Nesterov.

Distribution: Russia: European Part, Urals, Western and Eastern Siberia, Far East. - Europe, North Africa, Turkey, Kazakhstan, Mongolia, China, Korean Peninsula, Japan.

\section{Ectemnius (Metacrabro) spinipes (A. Morawitz, 1866)}

Irkutsk Province. 1 ㅇ Bolshoje Goloustnoje 24.06.2011, leg. Liebig. 2 ㅇ ㅇ Sarma 16./18.07.2007, leg. Liebig.

Primorskii Territory. $1 \sigma^{\star}$ Poshiga 20.06.2014, leg. Liebig.

Sakhalin. $1 \sigma^{*}$ Korsakov (Ptitche Lake) 12.08.1975, leg. Nesterov. $2 \sigma^{\star} o^{\star}, 2$ 우 우 Lugovoje 22.07.1980, leg. Nesterov. $2 \sigma^{\star} \sigma^{\star}, 1$ क Novoaleksandrovsk (without date), leg. Nesterov.

Distribution: Russia: European Part, Urals, Western and Eastern Siberia, Far East. - Europe, Azerbaijan, Turkey, Turkmenistan, Kazakhstan, Mongolia, China, Korean Peninsula, Japan.

Ectemnius (Yanonius) martjanowi (F. MorawITz, 1892)

Sakhalin. $1 \sigma^{\star}$ Lugovoje 22.07.1980, leg. Nesterov. $2 \sigma^{\star} \sigma^{\star}$, 1 ㅇ Novoaleksandrovsk (without date), leg. Nesterov.

Distribution: Russia: Western and Eastern Siberia, Far East. - Mongolia, China, Korean Peninsula, Japan, India.

\section{Gorytes aino TSUNEKI, 1963}

Primorskii Territory. 1 i Saratovka 19.06.2014, leg. Liebig.

Distribution: Russia: Eastern Siberia, Far East. - China, Japan.

\section{Gorytes albidulus (LEPELETIER, 1832)}

${ }^{\star}$ Irkutsk Province. 1 ơ, 1 ㅇ Sarma 14.07./28.06.2006, leg.

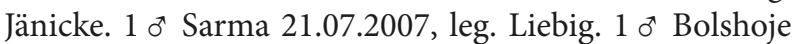
Goloustnoje 20.06.2011, leg. Liebig.

Distribution: Russia: European Part, Western Siberia (Altai). - Europe, North Africa, Azerbaijan, Turkey, Iran, Uzbekistan, Kyrgyzstan, Kazakhstan, Mongolia. New record for Eastern Siberia.

Remark: First recorded in Siberia in the Altai Mountains (BAgHiRov 2011). The next nearest records are in Mongolia (Zavchan Aimak, Chövsgöl Aimak) (TsuneKI 1971). Chövsgöl Aimak is located south of Zabaikalskii Territory. 


\section{Gorytes ambiguus HANDLIRSCH, 1888}

Irkutsk Province. $10^{\star}, 2$ 우 Bolshoje Goloustnoje 14.-25.06.2011, leg. Liebig.

Distribution: Russia: Western and Eastern Siberia, Far East. - Tajikistan, Uzbekistan, Kazakhstan, Mongolia, China.

\section{Gorytes laticinctus koreanus (LePELETIER, 1832)}

${ }^{\star}$ Irkutsk Province. $2 \sigma^{\star} \sigma^{\star}$ Ust-Ordynski (steppe) 27.06. 2011, leg. Liebig.

Distribution: Russia: Eastern Siberia, Far East. - Korean Peninsula, Japan.

\section{Gorytes neglectus HANDLIRSCH, 1895}

(Figs. 2e, 2f)

Buryat Republic. $6 \sigma^{\star} \sigma^{\star}, 1$ \& East-Sajan, Bank of Black Irkut 30.06.2012, leg. Liebig. $3 \sigma^{\top} o^{\star}, 5$ 우 우 East-Sajan, Mondy, 30 km NW 02.-13.07.2012, leg. Liebig.

Primorskii Territory. 1 i Kavalerovo 26.06.2014, leg. Liebig. 1 우 Poshiga 21.06.2014, leg. Liebig.

Distribution: Russia: European Part, Western and Eastern Siberia, Far East. - Europe, Turkey, Kazakhstan, Korean Peninsula.

\section{Gorytes pieli YASUMATSU, 1943}

${ }^{\star}$ Irkutsk Province. 1 o Bolshoje Goloustnoje 20.06.2011, leg. Liebig.

Distribution: Russia: Eastern Siberia, Far East. - Mongolia, China, Korean Peninsula.

\section{Gorytes procrustes HANDLIRSCH, 1888}

${ }^{\star}$ Irkutsk Province. $4 \sigma^{\star} o^{\star}$ Bolshoje Goloustnoje 20.-25. 06.2011, leg. Liebig. 1 ๙ $^{\star}$ Ust-Ordynski (steppe) 27.06.2011, leg. Liebig.

Distribution: Russia: European Part, Urals, Western Siberia (Altai, Baghirov 2011). - Europe, Georgia, Turkey, Kazakhstan. New record for Eastern Siberia.

\section{Gorytes quadrifasciatus (FABRICIUS, 1804)}

Irkutsk Province. $1 \sigma^{\star}, 2$ 우 우 Sarma 24.07.2007, leg. Liebig, det. Schmid-Egger. $7 \sigma^{\top} \sigma^{\top}$ Ust-Ordynski (steppe) 27.06.2011, leg. Liebig.

Distribution: Russia: European Part, Urals, Western and Eastern Siberia, Far East. - Europe, North Africa, Turkey, Kyrgyzstan, Kazakhstan, Mongolia, Korean Peninsula.

\section{Gorytes quinquecinctus (FABRICUS, 1793)}

Irkutsk Province. $1 \sigma^{x}, 1+$ Bolshoje Goloustnoje 20./25.06.2011, leg. Liebig.

Distribution: Russia: European Part, Urals, Western and Eastern Siberia. - Europe, North Africa, Azerbaijan, Turkey, Syria, Iran, Uzbekistan, Kyrgyzstan, Kazakhstan.

\section{Gorytes quinquefasciatus (PANZER, 1798)}

Irkutsk Province. $10 \sigma^{x} \sigma^{x}, 3$ 우 우 Bolshoje Goloustnoje 20.-25.06.2011, leg. Liebig. $1 \sigma^{\star}, 1$ ㅇ Ust-Ordynski (steppe) 27.06.2011, leg. Liebig.

Primorskii Territory. $2 \sigma^{*} \sigma^{*}$ Poshiga 20.06.2014, leg. Liebig.

Distribution: Russia: European Part, Urals, Western and Eastern Siberia, Far East. - Europe.

\section{Gorytes sulcifrons mongolicus TSUNEKI, 1971}

Irkutsk Province. 3 우 우 Sarma 18./21.07.2007, leg. Liebig.

Distribution: Russia: Western and Eastern Siberia. Azerbaijan, Kazakhstan, Mongolia, China.

Remark: The identification of the $o+9$ is confirmed by comparison with the original description of TsUNEKI (1971).

\section{Lestica alata (PANzer, 1797)}

Irkutsk Province. $4 o^{x} o^{x}, 1$ ㅇ Bolshoje Goloustnoje 20.-25.06.2011, leg. Liebig. $1 \sigma^{\star}$ Ust-Ordynski (steppe) 27.06.2011, leg. Liebig.

Zabaikalskii Territory. $1 \sigma^{*}, 1$ ㅇ Daurskij Reserve 24.07.1989, leg. Golovushkin.

Distribution: Russia: European Part, Urals, Western and Eastern Siberia, Far East. - Europe, North Africa, Turkey, Kyrgyzstan, Kazakhstan, Mongolia, China, Korean Peninsula, Japan, North India.

\section{Lestica camelus (Eversmann, 1849)}

Irkutsk Province. $50^{\star} o^{\star}, 5$ 우 우 Bolshoje Goloustnoje 19.-25.06.2011, leg. Liebig. 1 o Bolshoje Goloustnoje 18.07.2012, leg. Floßmann. $3 o^{\star} o^{\star}$ Sarma 18./21.07.2007, leg. Liebig.

Primorskii Territory. $5 \sigma^{x} \sigma^{x}, 2$ 우 Poshiga 20.-22.06. 2014, leg. Liebig.

Sakhalin. $10^{\top}$ Korsakov (Ptitche Lake) 12.08.1975, leg. Nesterov. $7 o^{\top} o^{\top}$ Lugovoje 22.07.1980, leg. Nesterov. 1 \% Novoaleksandrovsk, leg. Nesterov. 
Distribution: Russia: European Part, Urals, Western and Eastern Siberia, Far East. - Turkey, Kazakhstan, Mongolia, China, Korean Peninsula, Japan.

\section{Lestica clypeata (SCHREBER, 1759)}

Irkutsk Province. $2 \sigma^{\star} \sigma^{\star}, 2$ ㅇ ㅇ Sarma 16.-21.07.2007, leg. Liebig.

Distribution: Russia: European Part, Urals, Western and Eastern Siberia, Far East. - Europe, North Africa, Georgia, Turkey, Syria, Lebanon, Israel, Iraq, Iran, Turkmenistan, Uzbekistan, Kyrgyzstan, Kazakhstan.

\section{Lestica collaris (MATSUMURA, 1912)}

Primorskii Territory. $2 \sigma^{\top} \sigma^{\star}$ Dalnegorsk 24.06.2014, leg. Liebig. $1 \sigma^{\star}$ Poshiga 21.06.2014, leg. Liebig. $1 \sigma^{\star}$ Ussuriskij Zapovednik 28.08.1999, leg. Mattes.

Distribution: Russia: Far East. - Korean Peninsula, Japan.

\section{Lestica reiteri (KoHL, 1915)}

Primorskii Territory. 1 ơ Lazo 22.08.1999, leg. Mattes.

Distribution: Russia: Far East. - Korean Peninsula, Japan.

\section{Lestica subterranea ochotica (A. Morawitz, 1866)}

${ }^{\star}$ Irkutsk Province: 3 우 Sarma 16.-24.07.2007, leg. Liebig.

Distribution: Russia: Western and Eastern Siberia, Far East. - Mongolia, China.

\section{Lindenius panzeri (VANDER LINDEN, 1829)}

Irkutsk Province. $2 \sigma^{x} o^{x}, 2$ 우 Bolshoje Goloustnoje 20.-25.06.2011, leg. Liebig. 1 o , 1 क Sarma 17./21.07.2007, leg. Liebig.

Distribution: Russia: European Part, Urals, Western and Eastern Siberia, Far East. - Europe, North Africa, Turkey, Iran, Afghanistan, Turkmenistan, Uzbekistan, Kazakhstan, Mongolia, China.

\section{Mellinus crabroneus (THUNBERG, 1791)}

Irkutsk Province. $5 \sigma^{\star} o^{\star}$ Ust-Ordynski (steppe) 27.06. 2011, leg. Liebig.

Distribution: Russia: European Part, Urals, Western and Eastern Siberia, Far East. - Europe, Turkey, Kyrgyzstan, Kazakhstan, Mongolia, China, Korean Peninsula.

\section{Mimesa equestris (FABRICIUS, 1804)}

Irkutsk Province. $10^{*}$ Bolshoje Goloustnoje 25.06.2011, leg. Liebig.

Distribution: Russia: European Part, Urals, Western and Eastern Siberia, Far East. - Europe, Turkey, Kazakhstan, Korean Peninsula.

\section{Mimesa lutaria (FABRICIUS, 1787)}

Irkutsk Province. 15 ㅇ ㅇ Sarma 17.-24.07.2007, leg. Liebig. $10^{\star}$ Ust-Ordynski (steppe) 27.06.2011, leg. Liebig.

Distribution: Russia: European Part, Urals, Western and Eastern Siberia, Far East. - Europe, Turkey, Uzbekistan, Kyrgyzstan, Kazakhstan, Mongolia, China, Korean Peninsula, Japan, North America.

\section{Mimumesa dahlbomi (WeSMAEL, 1852)}

Primorskii Territory. $10^{\star}$ Anisimovka, Litovka Mt. 08.06.1993, leg. Taeger.

Distribution: Russia: European Part, Urals, Western and Eastern Siberia, Far East. - Europe, Turkey, Kyrgyzstan, Kazakhstan, Mongolia, China, Korean Peninsula, Japan.

\section{Miscophus bicolor JURINE, 1807}

Irkutsk Province. 1 o Sarma 21.07.2007, leg. Liebig, det. Schmid-Egger. 1 đ 1 , 1 ㅇ Bolshoje Goloustnoje 22.06./ 24.06.2011, leg. Liebig.

Distribution: Russia: European Part, Urals, Western and Eastern Siberia, Far East. - Europe, North Africa, Turkey, Syria, Iran, Kazakhstan, Mongolia, Korean Peninsula.

\section{Nysson dimidiatus JURINE, 1807}

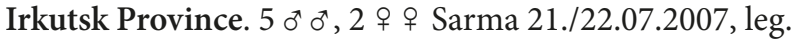
Liebig.

Distribution: Russia: European Part, Western and Eastern Siberia, Far East. - Europe, Armenia, Central Asia, Kazakhstan, Mongolia.

\section{Nysson interruptus (FABRICIUS, 1798)}

${ }^{\star}$ Irkutsk Province: 1 ㅇ Bolshoje Goloustnoje 20.06.2011, leg. Liebig. 2 ㅇ ㅇ Sarma 18./21.07.2007, leg. Liebig.

Distribution: Russia: European Part, Urals, Western Siberia. - Europe, North Africa, Turkey, Israel, Armenia, Kazakhstan. New record for Eastern Siberia. 


\section{Nysson maculosus (GMeLIN, 1790)}

Irkutsk Province. $1 \sigma^{x}, 1 \%$ Bolshoje Goloustnoje 20./25.06.2011, leg. Liebig. 1 o $^{\pi} 4$ 우 우 Sarma 18.-23. 07.2007, leg. Liebig.

Distribution: Russia: European Part, Urals, Western and Eastern Siberia, Far East. - Europe, North Africa, Caucasus, Turkey, Tajikistan, Uzbekistan, Kazakhstan, Mongolia, China, Korean Peninsula.

\section{Nysson spinosus (Forster, 1771)}

Khabarovsk Territory. 1 i Boitsovo 26.05.1993, leg. Taeger.

Primorskii Territory. $1 \sigma^{\star}, 1$ \& Bonevurovo 10.06.1993, leg. Taeger. 2 ㅇ ㅇ Anisimovka 07.06.1993, leg. Taeger. 3 우 ㅇ Ryazanovka 16.06.1993, leg. Taeger, 1 ㅇ Tikhoye nr. Razdolnoye 19.06.1993, leg. Taeger.

Distribution: Russia: European Part, Western and Eastern Siberia, Far East. - Europe, Azerbaijan, Turkey, Iran, Kazakhstan, Japan.

\section{Oxybelus haemorrhoidalis OLIVIER, 1812}

*Irkutsk Province: $11 o^{\star} o^{\star}, 3$ 우 우 Sarma 18.-24.07.2007, leg. Liebig.

Distribution: Russia: European Part, Urals, Western Siberia, Far East. - Europe, North Africa, Turkey, Syria, Israel, Iran, Afghanistan, Turkmenistan, Uzbekistan, Kyrgyzstan, Kazakhstan, Mongolia, China, Korean Peninsula, Japan. New Record for Eastern Siberia.

\section{Oxybelus trispinosus (FABRICIUs, 1787)}

Primorskii Territory. $1 \sigma^{\star}$ Anisimovka 02.07.2014, leg. Liebig. $1 \sigma^{\star}$ Saratovka 19.06 .2014 , leg. Liebig. $4 \sigma^{\star} \sigma^{\star}$ Kalinovka 29.06.2014, leg. Liebig.

Distribution: Russia: European Part, Urals, Western and Eastern Siberia, Far East. - Europe, North Africa, Turkey, Kazakhstan.

\section{Oxybelus uniglumis (LINNÉ, 1758)}

Irkutsk Province. 1 ㅇ Sarma 22.07.2007, leg. Liebig.

Distribution: Russia: European Part, Urals, Western and Eastern Siberia, Far East. - Europe, North Africa, Turkey, Tajikistan, Kyrgyzstan, Kazakhstan, Mongolia, North America.

\section{Passaloecus borealis DAHLBOM, 1844}

Primorskii Territory. 1 ㅇ Dalnegorsk 25.06.2014, leg. Liebig.
Distribution: Russia: European Part, Western and Eastern Siberia, Far East. - Europe, Kazakhstan, North America.

\section{Passaloecus monilicornis DaHLBOM, 1842}

Irkutsk Province. 2 đ ๙ $^{\star}$ Bolshoje Goloustnoje 25.06.2011, leg. Liebig. $1 \sigma^{\star}$ Ust-Ordynski (steppe) 26.06.2011, leg. Liebig.

${ }^{\star}$ Buryat Republic. 1 ㅇ East-Sajan, Mondy, $30 \mathrm{~km} \mathrm{NW}$ 10.07.2012, leg. Liebig.

Distribution: Russia: European Part, Urals, Western and Eastern Siberia, Far East. - Europe, Kazakhstan, Mongolia, China, Korean Peninsula, Japan, North America.

\section{Pemphredon inornata SAY, 1824}


NW 29.06.-13.07.2012, leg. Liebig.

Distribution: Russia: European Part, Urals, Western and Eastern Siberia, Far East. - Europe, North Africa, Turkey, Central Asia, Kazakhstan, Mongolia, China, Korean Peninsula, Japan, North America.

\section{Pemphredon lethifer (SHUCKARD, 1837)}

${ }^{\star}$ Buryat Republic. $6 o^{\star} o^{\star}, 8$ ㅇ ㅇ East-Sajan, Mondy, $30 \mathrm{~km}$ NW 28.06.-12.07.2012, leg. Liebig.

Distribution: Russia: European Part, Urals, Western Siberia, Far East. - Europe, North Africa, Azerbaijan, Turkey, Israel, Iraq, Iran, Tajikistan, Uzbekistan, Kyrgyzstan, Kazakhstan, Mongolia, China, Korean Peninsula, Japan, North America. New record for Eastern Siberia.

Remark: Distribution not certain, because in most cases P. lethifer und P. fabricii (MüLLER, 1911) have not been distinguished (see JАсОвS 2007).

\section{Pemphredon lugens DaHLBOM, 1842}

Irkutsk Province. $10 \sigma^{\star} \sigma^{*}, 1+$ Bolshoje Goloustnoje 12.-19.06.2011, leg. Liebig.

${ }^{\star}$ Buryat Republic. $1 \sigma^{\star}$ East-Sajan, Mondy, $30 \mathrm{~km} \mathrm{NW}$ 08.07.2012, leg. Liebig.

Distribution: Russia: European Part, Urals, Western and Eastern Siberia, Far East. - Europe, Turkey, Kazakhstan.

\section{Pemphredon lugubris (FABRICIUS, 1793)}

Irkutsk Province. 1 ㅇ Bolshoje Goloustnoje 23.06.2011, leg. Liebig. 
Distribution: Russia: European Part, Urals, Western and Eastern Siberia, Far East. - Europe, Turkey, Iran, Uzbekistan, Kyrgyzstan, Kazakhstan, Mongolia, China, Korean Peninsula, Japan, North America.

\section{Pemphredon montana DahlBom, 1844}

Irkutsk Province. $8 o^{\star} o^{\star}$ Bolshoje Goloustnoje 13.16.06.2011, leg. Liebig.

Primorskii Territory. 1 ㅇ Anisimovka 01.07.2014, leg. Liebig.

Distribution: Russia: European Part, Urals, Eastern Siberia, Far East. - Europe, Kazakhstan, China, Korean Peninsula, Japan, North America.

\section{Pemphredon mortifer VALKEILA, 1972}

Irkutsk Province. 1 ㅇ Bolshoje Goloustnoje 19.06.2011, leg. Liebig.

Distribution: Russia: European Part, Urals, Eastern Siberia, Far East. - Europe, Azerbaijan, Turkey, Korean Peninsula, Japan, North America.

\section{Philanthus hellmanni (EvERSMANN, 1849)}

Irkutsk Province. 1 \& Sarma 24.07.2007, leg. Liebig, det. Schmid-Egger.

Distribution: Russia: Western and Eastern Siberia, Far East. - Tajikistan, Kazakhstan, Mongolia, China.

\section{Pison strandi YASUMATSU, 1935}

Primorskii Territory. $1 \sigma^{\star}$ Kalinovka 29.06.2014, leg. Liebig.

Distribution: Russia: Far East. - Korean Peninsula, Japan.

Psen ater (OLIVIER, 1792)

Irkutsk Province. $1 \sigma^{\star}$ Bolshoje Goloustnoje 25.06.2011, leg. Liebig. $1 \sigma^{\star}$ Ust-Ordynski (steppe) 27.06.2011, leg. Liebig.

Distribution: Russia: European Part, Urals, Western and Eastern Siberia, Far East. - Europe, Turkey, Uzbekistan, Kazakhstan, Mongolia, China, Korean Peninsula, Japan.

\section{Psenulus anomoneurae (YASUMATSU, 1938)}

Primorskii Territory. 1 ㅇ Anisimovka 07.06.1993, $10^{\star}$ Anisimovka 08.06.1993, leg. Taeger.

Distribution: Russia: Far East. - Korean Peninsula, Japan.

\section{Psenulus pallipes (PANZER, 1798)}

Irkutsk Province. 1 o, 2 우 우 Sarma 21.-22.07.2007, leg. Liebig.

Distribution: Russia: European Part, Urals, Western and Eastern Siberia, Far East. - Europe, Armenia, Turkey, Iran, Kazakhstan, China, Korean Peninsula, Japan, North America.

Remark: Distribution not always certain, P. pallipes may be confused with P. chevrieri (TournIER, 1889) (see SCHMID-EgGER 2016).

\section{Rhopalum laticorne (TSUNEKI, 1947)}

Primorskii Territory. $10^{\star}$ Dalnegorsk 24.06.2014, leg. Liebig.

Distribution: Russia: Eastern Siberia, Far East. - China, Korean Peninsula.

Stizus perrisi Dufour, 1838

(Figs. 2g, 2h)

Primorskii Territory. 10 o $^{\star}, 13$ 우 아 Kalinovka 27-28. 06.2014, leg. Liebig.

Distribution: Russia: European Part, Urals, Western and Eastern Siberia, Far East. - Europe, Armenia, Turkey, Uzbekistan, Kyrgyzstan, Kazakhstan, China, Korean Peninsula, Japan.

Remark: Larra pulcherrima F. SMITH, 1856 is synonymized by Nemкov (2012b) with Stizus perrisi.

Tachytes panzeri (Dufour, 1841)

Irkutsk Province. $1 \sigma^{\star}, 3$ 우 Ust-Ordynski (steppe) 26.-27.06.2011, leg. Liebig.

Distribution: Russia: European Part, Urals, Western and Eastern Siberia. - Europe, North Africa, Turkey, Israel, Iran, Turkmenistan, Tajikistan, Uzbekistan, Kazakhstan.

\section{Trypoxylon figulus (LINNÉ, 1758)}

Irkutsk Province. $1 \sigma^{x}, 2$ 우 Bolshoje Goloustnoje 13./19.06.2011, leg. Liebig, Jänicke.

Buryat Republic. $8 \sigma^{\star} \sigma^{\star}, 4$ 우 우 East-Sajan, Mondy, $30 \mathrm{~km}$ NW 29.06.-14.07.2012, leg. Liebig.

Primorskii Territory. 1 ㅇ Poshiga 22.06.2014, leg. Liebig.

Distribution: Russia: European Part, Urals, Western and Eastern Siberia, Far East. - Europe, North Africa, Turkey, Tajikistan, Uzbekistan, Kyrgyzstan, Kazakhstan, Mongolia, China, Japan, North America. 


\section{Trypoxylon frigidum cornutum GUSSAKOVSKIJ, 1932}

Primorskii Territory. $1 \sigma^{\star}, 3$ ㅇ ㅇ Poshiga 21.06.2014, leg. Liebig.

Distribution: Russia: Western and Eastern Siberia, Far East. - Korean Peninsula, Japan.

\section{Trypoxylon koreanum TsUNEKI, 1956}

Primorskii Territory. 1 \& Novonezhino 30.06.2014, leg. Liebig.

Distribution: Russia: European Part, Far East. - China, Korean Peninsula.

\section{Trypoxylon ussuriense KaZENAS, 1980}

Primorskii Territory. 1 ㅇ Kalinovka 27.06.2014, leg. Liebig.

Distribution: Russia: Far East.

\section{Tzustigmus rhinocerus (BuDRYs, 1987)}

Primorskii Territory. 1 ㅇ Mezhdurechje 28.05.1993, leg. Taeger.

Distribution: Russia: Far East. - Korean Peninsula.

\section{Not determined taxa}

\section{Ammoplanus GIRAUD, 1869}

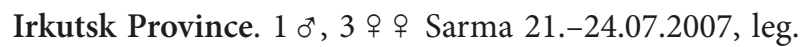
Liebig.

Remark: These specimens could not be identified with the keys by MARSHAKov (1979) and BoučEK (2001).

\section{Nitela LATREILLE, 1809}

Irkutsk Province. 1 o $^{\text {T }}$ Bolshoje Goloustnoje 19.06.2011, leg. Liebig.

Remark: This specimen could not be identified with the key by Gayubo \& Felton (2000).

\section{Harpactus SHUCKARD, 1837}

Buryat Republic. $1 \sigma^{\star}$ East-Sajan, Mondy, $30 \mathrm{~km} \mathrm{NW}$ 13.07.2012, leg. Liebig.

Remark: This specimen could not be identified with the key by Nemkov (1996).

\section{Results}

Seven species are recorded first for Eastern Siberia: Crossocerus (Crossocerus) tarsatus (SHUCKARD, 1837), Diodontus handlirschi KoHL, 1888, Gorytes albidulus (LePeletier, 1832), Gorytes procrustes Handlirsch, 1888, Nysson interruptus (FABRICIUs, 1798), Oxybelus haemorrhoidalis OLIVIER, 1812 and Pemphredon lethifer (SHUCKARD, 1837). Ectemnius guttatus (VANDER LiNDEN, 1829 ) is new for the Far East.

There are some new regional records:

Eastern Siberia, Tuva Republic: Crossocerus (Crossocerus) yasumatsui (TsUNEKI, 1947).

Eastern Siberia, Irkutsk Province: Podalonia asiatica Danilov, 2017, Cerceris pedetes KoHL, 1887, Diodontus dziuroo Tsuneki, 1972, Diodontus gegen Tsuneki, 1972, Ectemnius (Clytochrysus) sexcinctus (FABricius, 1775), Gorytes albidulus (LEPELETIER, 1832), Gorytes laticinctus koreanus Handlinsch, 1888, Gorytes pieli YAsumatsu, 1943, Gorytes procrustes HANDLIRSCH, 1888, Lestica subterranea ochotica (A. MORAWITZ, 1866), Nysson interruptus (FABRICIUS, 1798), Oxybelus haemorrhoidalis Olivier, 1812

Eastern Siberia, Buryat Republic: Podalonia alpina (КонL, 1888), Crabro (Anothyreus) maeklini A. Morawitz, 1866, Crossocerus (Blepharipus) leucostoma (Linné, 1758), Crossocerus (Crossocerus) elongatulus (VANDER Linden, 1829), Crossocerus (Crossocerus) kohli (BIschoff, 1921), Crossocerus (Crossocerus) tarsatus (SHUCKARD, 1837), Crossocerus (Crossocerus) varus LePeletier \& BRULlé, 1835, Diodontus collaris Tsuneki, 1972, Diodontus handlirschi Koнl, 1888, Diodontus medius DAнlвом, 1844, Dryudella pinguis (DАнLВом, 1832), Passaloecus monilicornis DAHLBOM, 1842, Pemphredon lethifer (ShuCKARD, 1837) and Pemphredon lugens DAнLвом, 1842.

Far East, Sakhalin: Ectemnius (Ectemnius) guttatus (VANDER Linden, 1829).

\section{Acknowledgement}

The authors are indebted to Stephan Schmidt (Munich), Christian Schmid-Egger (Berlin) and Malte Jänicke (Eisenberg) for the loan of material. We thank Christian Schmid-Egger (Berlin) and Valeriy M. Loktionov (Vladivostok) for critical reading the manuscript and critical directions. Andrew D. Liston (Müncheberg) corrected English. Wolf-Harald Liebig would like to thank Prof. Sergey Pyjyanov (Irkutsk) for the logistic support of the expeditions to the Baikal region and the Buryat Republic. 
Literatur

BAghiRov, R. T. 2011: Fauna of the digger wasps (Hymenoptera: Sphecidae, Crabronidae) of the Altai Territory and Republic of Altai. - Entomologicheskoye Obozreniye 90: 138-158.

Belokobylskij, S. A. \& Lelej, A. S. 2017: Annotated Catalogue of the Hymenoptera of Russia. Volume I. Symphyta and Apocrita: Aculeata. - Proceedings of the Zoological Institute of the Russian Academy of Sciences. Suppl. 6: 1-477.

BoučEK, Z. 2001: Palaearctic species of Ammoplanus (Hymenoptera: Sphecidae). _ _ Lournal of _ Natural History 35: 849-929. - ihttps://doi.org/10.1080/ 00222930152123648

DANILŌ, Yu. N. $\overline{2} 0 \overline{1} \overline{4} \bar{a}$ : Review of Sphecidae wasps (Hymenoptera: Apoidea) of Siberia. Part I. List of species. - Euroasian Entomological Journal 13: 422-429.

Danilov, Yu. N. 2014b: Review of Sphecidae wasps (Hymenoptera: Apoidea) of Siberia. Part 2. Key to genera and species. - Euroasian Entomological Journal 13: 511-521.

DANILOv, YU. N. 2017: Taxonomic notes on Palearctic Podalonia Fernald, 1927 (Hymenoptera, Sphecidae), with descriptions of four new_species. - Zootaxa, 4320 (3): 554-570. - "http://dx.doi.org/10.11646/, zootaxa.4320.3.9.

DoLlfuss, H. 2010: A key to wasps of the genus Podalonia Fernald 1927 (Hymenoptera: Apoidea: Sphecidae) of the Old World. = Linzer Biologische Beiträge 42: 1241-1291. - https://www.zobodat.at// pdf/LBB_0042_2_1241-1291.pdf

Dollfuss, H. 2013 : Revision of the wasp genus Ammophila Kirby 1798 (Hymenoptera: Apoidea: Sphecidae) of the Palearctic Region and India. Linzer Biologische Beiträge 45: 383-564. www.zobodat.at/pdf/LBB_0045_1_0383-0564.pdf

GaYUBo, S. F. \& FELTON, J. 2000 : The European species of the genus Nitela Latreille, 1809 (Hymenoptera: Sphecidae). - Annales de la Société Entomologique de France (Nouvelle Série) 36: 291-313.

JACOBS, H.-J. 2007: Hymenoptera III. Die Grabwespen Deutschlands. Ampulicidae, Sphecidae, Crabronidae. Bestimmungsschlüssel. - In: BLANK, S. M. \& TAeger, A. (Hrsg.): Die Tierwelt Deutschlands und der angrenzenden Meeresteile nach ihren Merkmalen und nach ihrer Lebensweise. Begründet 1925 von Friedrich Dahl. 79. Teil. - Goecke \& Evers, Keltern: $1-207$.

КонL, F. F. 1906: Die Hymenopterengruppe der Sphecinen. III. Monographie der Gattung Ammophila W. Kirby (sens. lat. - Ammophilinae Ashmead). Abteilung A. Die Ammophilinen der paläarktischen Region. - Annalen des k.k. Naturhistorischen Hofmuseums 21: 228_382, pls. VII-XIII. - hittps://' www.zobodat.at/pdf/ANNA_21_0228-0382.pd f
KoHL, F. F. 1915: Die Crabronen (Hymenopt.) der paläarktischen Region. - Annalen des k.k. Naturhistorischen Hofmuseums Wien 29: 1-453, pl. I-XIV. - ihttps://www.zobodat.at/pdf/ANNA_29_0001-0453. pdit.

LeCLERCQ, J. 1974: Pour la connaissance des Crossocerus asiatiques du sous-genre Cuphopterus Morawitz (Hymenoptera Sphecidae Crabroninae). - Bulletin de la Société Royale des Sciences de Liège 43: 659-668.

Marshakov, V. G. 1979: Royushchiye osy rodov Protostigmus Turner i Ammoplanus Giraud (Hymenoptera, Sphecidae) fauny Mongolii i Sredney Azii. - Nasekomyie Mongolii 7: 362-374.

Nemkov, P. G. 1989: Royushchiye osy roda Gorytes (Hymenoptera, Sphecidae) Sibiri i Dal'nego Vostoka SSSR. - Zoologicheskiy Zhurnal 68: 66-73.

Nemkov, P. G. 1990: Royushchiye osy triby Gorytini (Hymenoptera, Sphecidae) fauny SSSR. Rody Gorytes Latreille, Pseudoplisus Ashmead, Kohlia HANDLIRSCH. - Entomologicheskoye Obozreniye 69: 675-690.

Nemkov, P. G. 1996: Royushchiye osy triby Gorytini (Hymenoptera, Sphecidae) fauny Rossii i sopredel' nykh stran. Rod Harpactus. - Zoologicheskiy Zhurnal 75: 1204-1213.

Nemкov, P. G. 2008: The digger wasps fauna (Hymenoptera: Sphecidae, Crabronidae) of the Asiatic part of Russia. - A. I. Kurentsov's Annual Memorial Meetings 19: 15-34.

Nemкоv, P. G. 2009: Annotated catalogue of digger wasps (Hymenoptera: Sphecidae, Crabronidae) of Asian part of Russia. - Dal'nauka, Vladivostok: 1-195.

Neмкоv, P. G. 2012a: Sektsiya Spheciformes - Royushchiye osy: 433-447. - In: Lelej, A. C. (ed.): Annotated catalog of insects of the Far East of Russia. Volume I. Hymenoptera. - Dal'nauka, Vladivostok: 1-635.

Nemkov, P. G. 2012b: Digger wasps of the genus Stizus LAtreille, 1802 (Hymenoptera, Crabronidae, Bembicinae) of the fauna of Russia and neighbouring countries. - Euroasian Entomological Journal 11: 55-62.

Nemkov, P. G.; Kazenas, V.L.; Budrys, E. R. \& Antropov, A. V. 1995: Superfam. Sphecoidea. 67. Fam. Sphecidae - Digger wasps: 368-480. - In: LeHr, P. A. (ed.): Key to the insects of Russian Far East in six Volumes. Vol. IV. Neuropteroides, Mecoptera, Hymenoptera. Part 1. - Nauka, Sankt Peterburg.

Schmid-Egger, C. 2002: Key and new records for the western Palearctic species of Gorytes LATREILLE 1804 with description of a new species (Hymenoptera, Sphecidae, Bembicinae). _ _ _Linzer_ _Biologische Beiträge 34: 167-190. - https://www.zobodat.at/pdf// LBB_0034 1_0167-0190.pdfi 

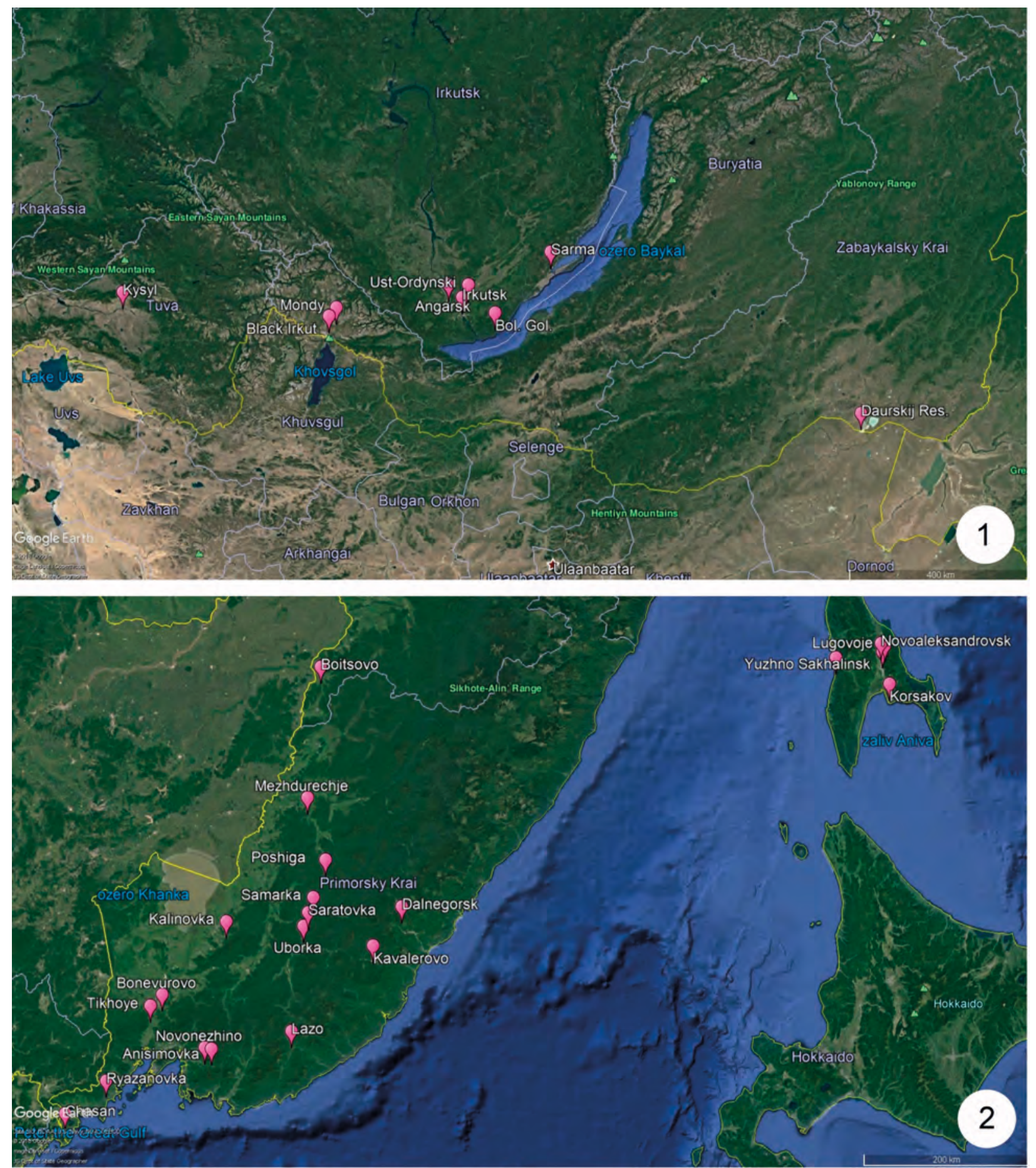

Fig. 1: Maps of collecting sites. Map 1: Collecting sites in Tuva, the Baikal area, Buryatia and Transbaikalia (Tchita Oblast). Map 2: Collecting sites in Primorye and Sakhalin.

Schmid-Egger, C. 2016: The Psenulus pallipes species group in Central Europe (Hymenoptera, Crabronidae). - Ampulex 8: 40-44.

SchmidT, K. 2000: Bestimmungstabelle der Gattung Cerceris Latreille, 1802 in Europa, dem Kaukasus, Kleinasien, Palästina und Nordafrika (Hymenoptera, Sphecidae, _ _ Philanthinae). _. Stapfia 71:1-325. - https://www.zobodat.at/pdf/ STAPFIA_0071_0001-0251.pdfi
TsuneKI, K. 1971: Ergebnisse der zoologischen Forschungen von Dr. Z. Kaszab in der Mongolei. 239. Sphecidae (Hymenoptera). I-II. - Acta Zoologica Academiae Scientiarum Hungaricae 17: 139-217. 

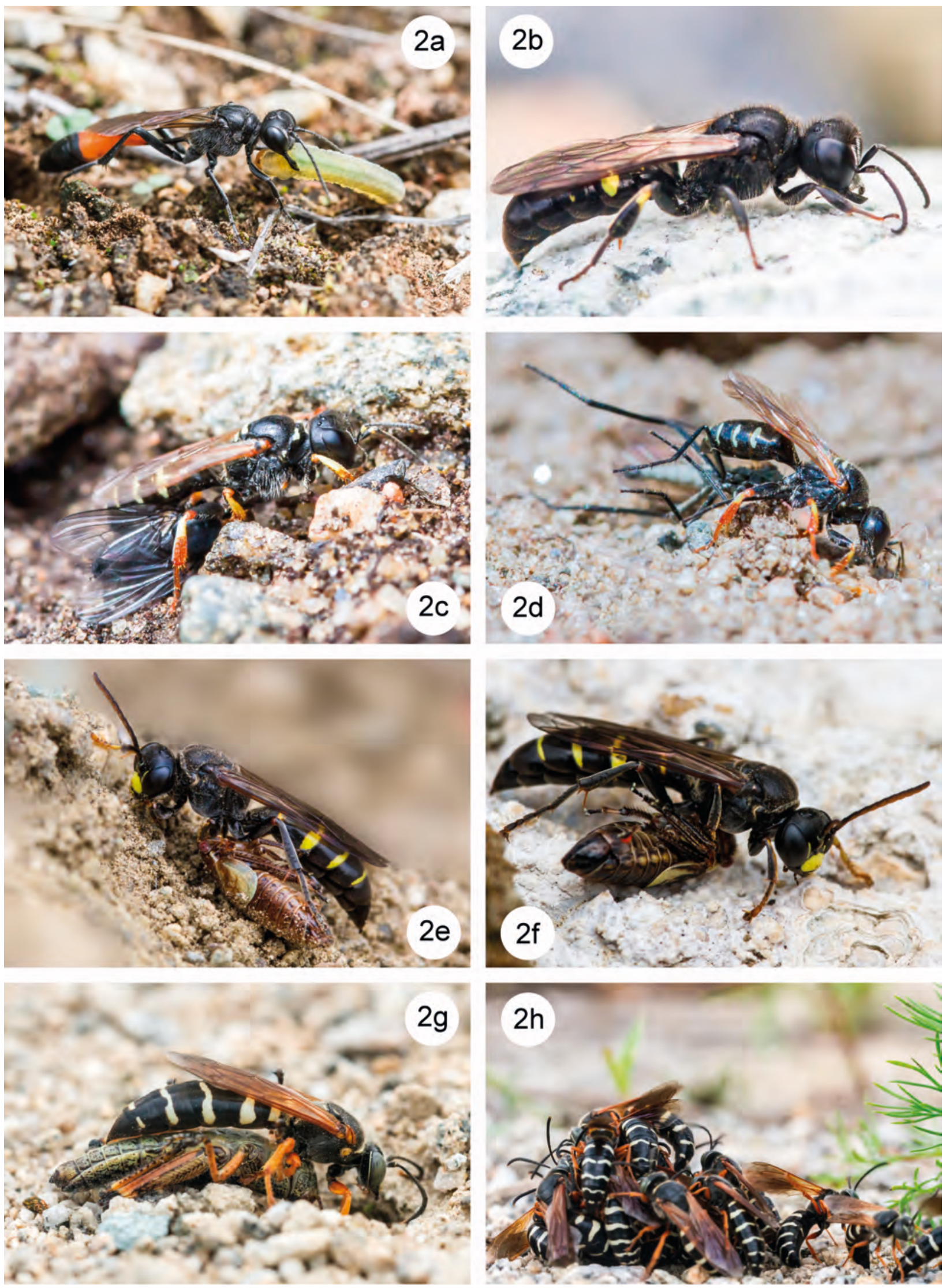

Fig. 2a-h: Digger wasps in action. 2a. Ammophila mongolensis of with prey. 2b. Crabro (Anothyreus) lapponicus o . 2c-d. Crabro (Anothyreus) maeklini + with prey. 2e-f. Gorytes neglectus + with prey. 2g. Stizus perrisi + with prey. 2h. Stizus perrisi $0^{\star} 0^{\top}$ are waiting for emergence of a ․ 